\title{
电刺激兔中脑中央灰质及其 邻近结构的镇痛作用
}

\author{
许冠苏起宝文 王金根
}

(上海生理研究所针麻组)

以前的工作表明, 针刺镇痛效应的中枢 传入途径主要是脊髓外侧索 ${ }^{[1]}$. 同时, 在脊 䯠、脑干和丘脑观察到了针刺抑制由伤害性 刺激引起的神经细胞放电 ${ }^{[2-4]}$. 现有的神经 解剖学和生理学资料证明, 脊髓前外侧索的 一部分上行纤维，投射到延脑内侧网状结构 的巨细胞核; 从此核发出的一些纤维经中央 灰质及其邻近的中央被盖束上行, 止于丘脑 的中央中核等内䯣板内核群 ${ }^{5,6]}$. 中央灰质及 其邻近结构在痛觉信息与深部感觉信息传递 中起着一定的作用 ${ }^{[7,8]}$.

电刺激该结构对痛反应的影响, 以前的 工作结果很不一致: 局部重复电刺激猫、猴 的中央灰质区, 可以引起动物㟫叫、坚毛、逃 跑和攻击等反应。在人, 电刺激中央灰质区 即出现恐惧和中线部位的弥散深部疼痛 ${ }^{[7-9]}$. 另一方面, 电刺激鼠、猫中央灰质边缘区可使 痛觉消失, 甚至在连续刺激情况下, 可进行剖 腹探查手术 ${ }^{[10-12]}$.

为了探讨针刺镇痛效应的中枢传递途 径, 本实验观察微弱电流刺激兔中脑中央灰 质及其邻近结构, 对痛反应的改变和对针刺 镇痛作用的影响.

\section{一、实验方法}

实验用 10 只体重为 $1.9-2.5$ 公斤的家 兔, 性别不分. 辐射热测痛阈方法是我们以 往所用的 ${ }^{[1]}$, 以 500 瓦、220 伏的光源, 经聚 焦后照射到兔鱟尖作为痛刺激. 从照射开始
到动物甩头逃避灼热这一段“反应时”，表示 动物的痛间。对每只兔, 先测试痛阈三次, 如 果稳定的话, 就选用此兔进行慢性埋藏电极 手术. 埋藏电极用直径为 0.2 毫米的不锈钢 丝, 外涂绝缘漆, 顶端剪成斜面, 两根电极平 行距离为 1 毫米, 上下相差 0.5 毫米. 电极末 端焊上导线, 焊接处用牙科水泥固定. 在戊 巴比妥钠麻醉下 (按 40 毫克/公斤剂量, 静脉 注射),切开头皮,用牙科钻在领骨一定部位钻 一小孔，借定位仪按 Sawyer 兔脑图谱 ${ }^{[13]}$, 将 双极电极插人中脑中央灰质及其邻近的被盖 区 $\left(\mathrm{P}_{8-10}\right.$ 平面 $)$, 用牙科水泥固定之. 术后第 四天开始实验。

实验过程:（1）每隔 5 分钟测痛阈一次， 一俟痛阈稳定, 即以 30 和 60 微安的 60 周/秒 正弦波电流刺激中央灰质及其邻近被盖一分 钟, 观察痛间的变化和恢复过程. 每一动物 的电刺激效应重复 4-6 次; (2)对一些动物, 以 60 周/秒的正弦波, 在 10-100 微安的电流 强度范围内, 测定不同强度电流刺激对痛间 的影响, 得出强度一痛阈变化曲线; (3)以对痛 间影响大的电流强度, 在 20-400 周/秒的频 率范围内, 测定不同的刺激频率对痛國的影 响, 得出频率一痛阈变化曲线; (4)在电针刺激 双侧足三里(电针参数为 60 周/秒, 波宽为 1 毫秒, 6 伏)的背景上, 测定电刺激中央灰质 及其邻近结构对针刺镇痛作用的影响.

本文 1973 年 11 月 3 日收到. 
表 1 刺激中央灰质及其邻近被盖区对痛國的影响

\begin{tabular}{|c|c|c|c|c|c|c|c|c|c|c|c|}
\hline \multirow{2}{*}{ 动物编号 } & \multirow{2}{*}{$\begin{array}{c}\text { 刺激强度 } \\
\text { (微安) }\end{array}$} & \multicolumn{2}{|r|}{ 中 央 } & \multicolumn{3}{|c|}{ 区 } & \multicolumn{5}{|c|}{ 中 央 被 盖 束 区 } \\
\hline & & (刺激前 $)$ & $\begin{array}{c}\text { 刺激时 } \\
\text { 秒均值) }\end{array}$ & 差 数 & $\begin{array}{c}\text { 痛阈提高 } \\
\%\end{array}$ & 效 应 & 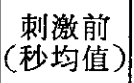 & 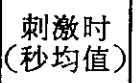 & 差 数 & $\begin{array}{c}\text { 痛阈提高 } \\
\%\end{array}$ & 效 应 \\
\hline \multirow{2}{*}{41} & 60 & 2.7 & 3.2 & 0.5 & 17.7 & 无 & 3.0 & 2.2 & -0.7 & -29.1 & 无 \\
\hline & 30 & 2.1 & 2.5 & 0.4 & 19.0 & 无 & 3.1 & 2.4 & -0.8 & -28.1 & 无 \\
\hline \multirow{2}{*}{42} & 60 & 3.3 & 3.0 & -0.3 & -8.8 & 无 & 4.6 & 3.9 & -0.7 & -16.5 & 无 \\
\hline & 30 & 4.2 & 3.9 & -0.3 & -3.3 & 无 & 4.7 & 4.3 & -0.4 & -7.9 & 无 \\
\hline \multirow{2}{*}{43} & 60 & 5.2 & 9.8 & 4.6 & 68.2 & 有 & 4.4 & 13.3 & 8.9 & 209.8 & 有 \\
\hline & 30 & 4.9 & 4.8 & -0.1 & -6.5 & 无 & 4.9 & 4.7 & -0.2 & -4.4 & 无 \\
\hline \multirow{2}{*}{44} & 60 & 3.9 & 3.5 & -0.4 & -13.7 & 无 & 3.9 & 4.0 & 0.1 & 2.5 & 无 \\
\hline & 30 & 3.1 & 3.2 & 0.1 & 3.3 & 无 & 4.5 & 4.5 & 0 & 0 & 无 \\
\hline \multirow{2}{*}{46} & 60 & 4.0 & 2.1 & -1.9 & -31.1 & 无 & 4.1 & 12.3 & 8.2 & 214.8 & 有 \\
\hline & 30 & 4.0 & 4.3 & 0.3 & 11.3 & 无 & 4.0 & 4.3 & 0.3 & 6.3 & 无 \\
\hline \multirow{2}{*}{47} & 60 & 5.2 & 8.4 & 3.2 & 61.3 & 有 & 4.1 & 8.8 & 4.7 & 114.9 & 有 \\
\hline & 30 & 5.2 & 6.1 & 0.9 & 16.8 & 无 & 3.5 & 6.0 & 2.5 & 78.4 & 有 \\
\hline \multirow{2}{*}{48} & 60 & 4.8 & 10.0 & 5.2 & 107 & 有 & 3.0 & 11.8 & 8.8 & 292.9 & 有 \\
\hline & 30 & 4.6 & 5.4 & 0.8 & 17.2 & 无 & 2.9 & 3.3 & 0.4 & 30.3 & 无 \\
\hline \multirow{2}{*}{49} & 60 & 3.5 & 8.8 & 5.3 & 151.4 & 有 & 4.7 & 9.8 & 5.1 & 109.5 & 有 \\
\hline & 30 & 3.3 & 4.3 & 1.0 & 30.2 & 无 & 4.8 & 5.4 & 0.6 & 14.3 & 无 \\
\hline \multirow{2}{*}{50} & 60 & 3.2 & 8.0 & 4.8 & 150 & 有 & 2.9 & 9.9 & 7.0 & 245.2 & 有 \\
\hline & 30 & 3.4 & 3.6 & 0.2 & 5.8 & 无 & 3.1 & 2.9 & -0.2 & -7.5 & 无 \\
\hline \multirow{2}{*}{51} & 60 & 5.0 & 9.7 & 4.7 & 106.5 & 有 & 4.3 & 10.6 & 6.7 & 146.5 & 有 \\
\hline & 30 & 4.9 & 5.0 & 0.1 & 2.0 & 无 & 4.5 & 5.1 & 0.6 & 13.3 & 无 \\
\hline
\end{tabular}

实验结束后，经埋藏电极通以微弱的阳 极电流. 用含 $1 \%$ 亚铁氯化钾的 $10 \%$ 福尔马 林溶液作心胜灌注, 取出脑组织, 再放在 $10 \%$ 福尔马林中固定 48 小时. 作 40 微米厚的冰 冻切片, 用硫莒染色, 显微镜检查可见到电极 尖端在脑组织中留下蓝色斑点.

\section{二、实验结果和讨论}

1. 电刺激中央灰质及邻近被盖对痛反应 的影响

在十只兔上的 20 个刺激点, 以方法 (1) 中所述的两种电流强度, 重复刺激 1 分钟, 观
察痛阈的变化，实验资料见表 1，从此表可 以看出,在 20 个刺激点中,有 13 个刺激点对 以 60 微安的电流强度重复刺激, 显示能明显 提高痛阈，如 46 号兔在刺激被盖区时，可以 使痛阈提高三倍多;有 7 个刺激点,无论是 60 微安或 30 微安的强度刺激, 对痛间无明显的 改变,如 41 号、42 号、43 号兔. 比较镇痛效 应时，发现中央灰质区刺激的镇痛效果不如 邻近被盖区刺激的镇痛效果明显. 组织学定 位见图 1. 从图 $1 \mathrm{~A}$ 看到,刺激中央灰质的背 侧及邻近被盖区，显示镇痛效应的有效点比 较集中在中脑的尾端 ( $\mathrm{P}_{10}$ 平面). 


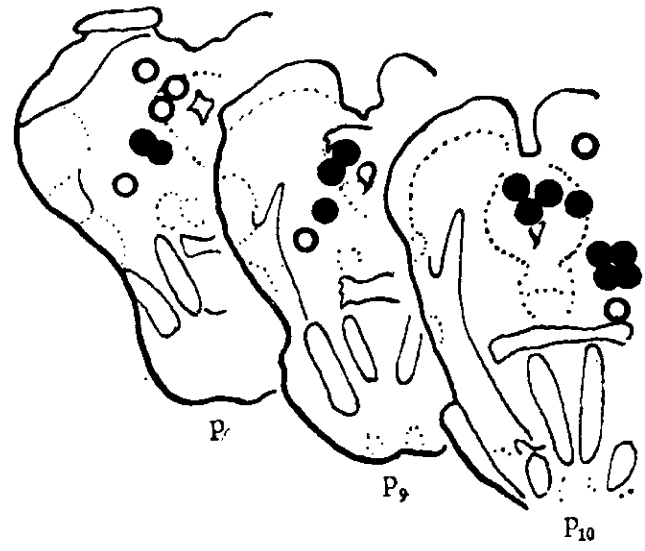

图 1A 电刺激中央灰质及邻近被盖对 痛反应的影响,组织学定位图 黑周有镇痛效应, 白圈无镇痛效应
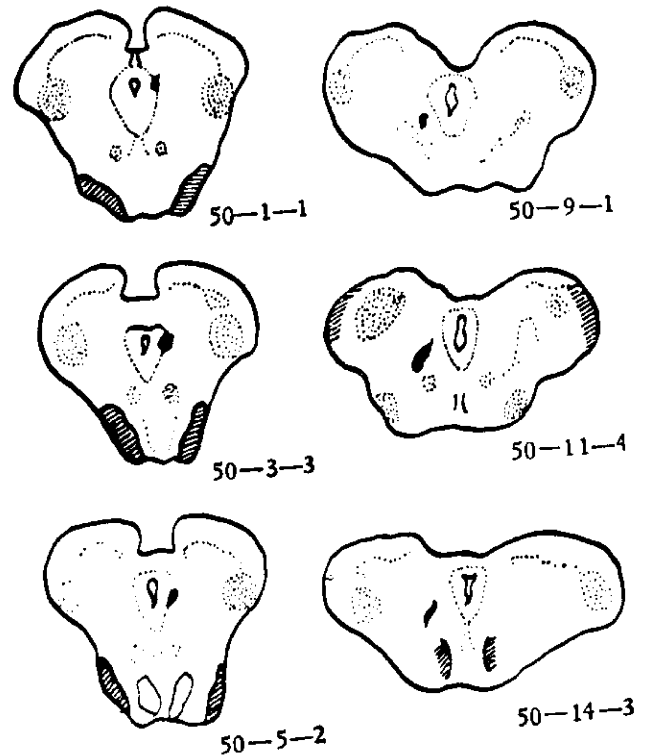

图 1B 50 号免刺激电极的组织学切片定位图 左侧示中央灰质区, 右侧示邻近被盖区

2. 刺激电流的参数与镇痛作用的关系

为了研究刺激电流的不同参数对痛反应 的影响, 我们在 5 例动物上测定了电流强度一 痛阈变化曲线和频率-痛國变化曲线. 图 2 是 48 号兔的电流强度-痛國变化曲线, 可以 看到, 刺激频率为 60 周/秒, 当电流强度低于 30 微安, 痛阈无明显变化; 而在 40一70 微安 范围, 即出现明显的镇痛作用; 当电流强度超 过 70 微安时,动物就出现逃跑和挣扎等痛反 应了. 图 3 是 50 号兔的频率一痛阈变化曲线, 可以看到, 刺激的电流强度固定在 60 微安,

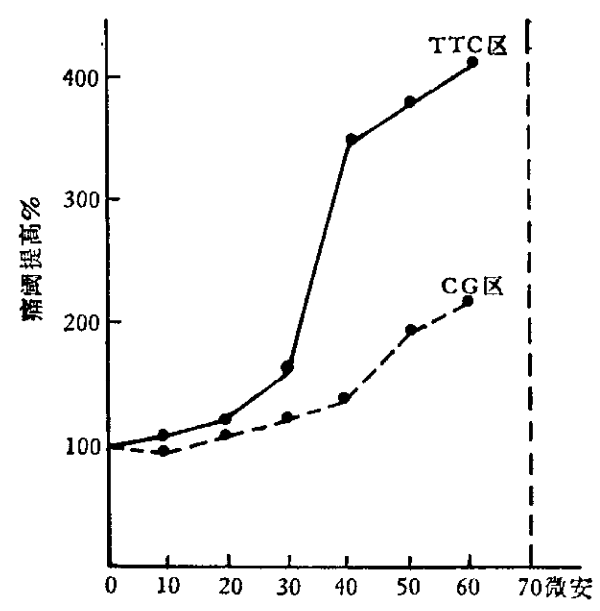

图 248 号兔的电流强度一痛阈变化曲线

TTC 示邻近被盖区, CG 示中央灰质区

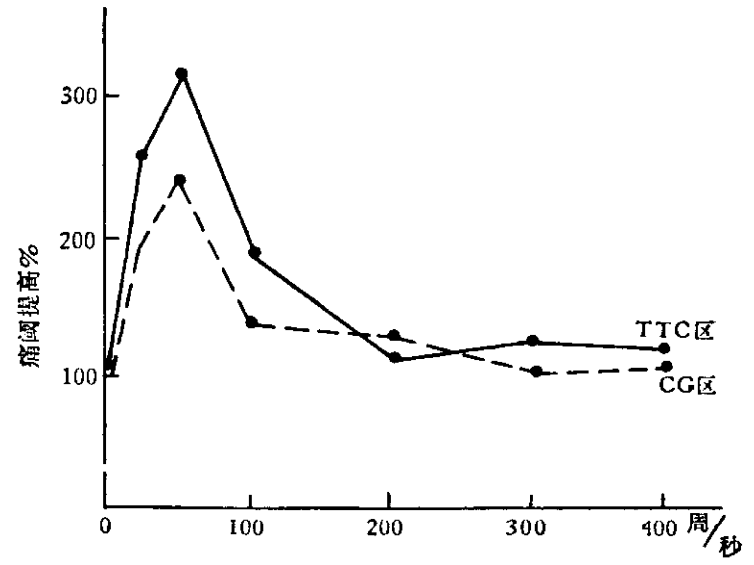

图 350 号兔的刺激电流频率一痛國变化曲线

当正弦波的频率在 20-100 周/秒范围变动 时,发现在 20 周/秒和 60 周/秒时,痛阈有明 显的提高; 当频率在 100-400 周/秒或更高 时,痛阈无明显变化. 通过上述的实验, 我们 认为，刺激中央灰质及邻近被盖产生的镇痛 作用，与刺激参数关系很大. 据此，我们推 测，以往有不少关于刺激该区的结果并不一 致的原因,可能是由于动物种类的不同,刺激 部位的微小差异，尤其是刺激参数的不同所 致.

3. 电针刺激背景上，刺激中央灰质及邻 近被盖对痛國的影响

关于刺激这些结构对电针镇痛效应的影 响, 我们对 6 例动物进行了初步观察. 图 4 是 50 号兔的结果. 看来,虽然局部刺激对电 


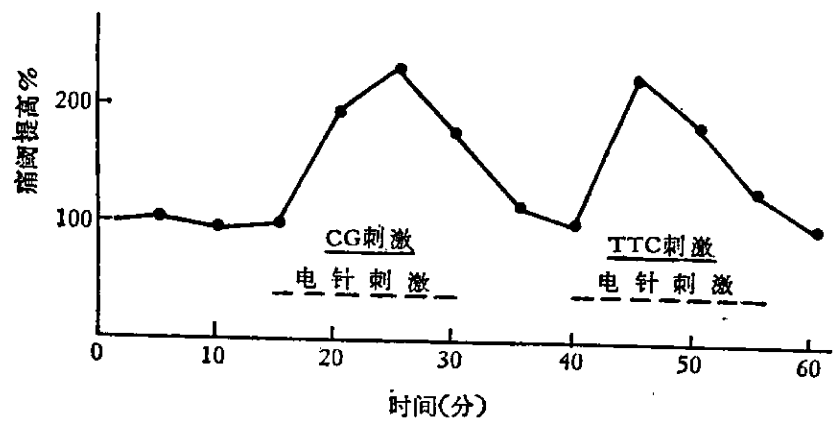

图 4 电针刺激背景上,刺激中央灰质及邻近 被盖对痛阈的影响

针镇痛效应的影响不够明显，但是它并没有 对抗电针镇痛的作用.

\section{参考资 料}

[1] 江振裕等,科学通报, 19 (1974), 1, 31 .

[2] 吴建屏等,科学通报, 18 (1973), 5, 238 .

[3] 上海中医学院等, 中华医学杂志, 1973, 3, 139-
143.

[ 4] 张香桐, 中国科学, 1973, 1, 28一-52.

[ 5 ] Mehler, W. R., Feferman, M. E. and Nauta, W. J. H., Brain, 83 (1960), 718-758.

[6] Nauta, W. J. H. and Kuypers, G. J. M., In Reticular Formation of the Brain, Ed. by Jasper, H. H. et al., Little, Brown, Boston. $1958,3-30$.

[ 7 ] Collins, W. F. and Randt, C. T., J. Neuraphysiol., 23 (1960), 47-53.

[ 8 ] Giuck, H., Becker, D. P. Nulsen, F. E. and Jane, J. A., J. Neurosurg., 30 (1969), 1-13.

[ 9 ] Nakao, H., Yoshida, M. and Sasaki, T., Jap. J. Physiol, 18 (1968), 462-470.

[10] Nashold, B. S., Wilson, W. P. and Slaughter, D. G., J. Neurosurg., 30 (1969), 14-24.

[11] Reynolds, D. V., Science, 164 (1969), 444445.

[12] Mayer, D. J. et al., Science, 174 (1971), 1351-1354.

[13] Sawyer, C. H., Everett, J. W. and Green, J. D., J. Comp. Neurol., 101 (1954), 801-824.

\title{
肉書杆菌毒素阻遏的大鼠神经肌肉 接头在降温后传递功能的恢复
}

\author{
施玉樑沈克飞 \\ (上海生理研究所)
}

肉毒杆菌毒素对胆碱能神经纤维传递的 阻遏作用被认为是不可逆的，毒素与其受体 的结合早在潜伏期的初期业已完 成 ${ }^{[1]}$. 迄今 为止，还没有任何一项措施能使离体神经肌 肉标本被肉毒毒素完全阻断传递的接头恢复 功能. 本文报道经 A 型肉毒杆菌毒素处理完 全麻㾇的接头在降低温度后的恢复.

实验用大白鼠膈神经膈肌标本. 标本制 备、保养及中毒条件同前 ${ }^{[2]}$, 标本以含 $\mathrm{A}$ 型肉 毒杆菌毒素的生理溶液(毒力 $1.7 \times 10^{4}$ 小鼠 最小致死量/毫升溶液)处理半小时。用充以 $3 M \mathrm{NaCl}$ 的玻璃微电极做细胞外记录, 微电 极尖端外径 2-3 $\mu$. 电生理观察从标本对间 接刺激不收缩时开始，电反应直接照相或以
生物用电子计算机叠加处理. 收缩描记按资 料 [3] 进行.

结 果

\section{一、收缩描记}

在我们的实验条件下, $37^{\circ} \mathrm{C}$ 时标本从用 A 型肉毒素处理到在记纹鼓上描记不到收缩 一般约经 60-70 分钟. 此时若给标本施以 间接重复刺激 (100 次/秒一-300 次/秒) 肌肉 仍能出现收缩, 但随着毒素作用的发展, 麻㾇 加深，不久这种对重复刺激的收缩反应也完 全消失. 此后, 若将标本溶液温度降低 10-

本文 1974 年 6 月 3 日收到. 